\title{
Transfer hydrogenolysis of aromatic alcohols using Raney catalysts and 2-propanol
}

\author{
Benjamin H. Gross ${ }^{1}$, Robert C. Mebane*, David L. Armstrong \\ Department of Chemistry, University of Tennessee at Chattanooga, Chattanooga, TN 37403-2598, USA
}

Received 9 February 2001; received in revised form 8 June 2001; accepted 10 June 2001

\begin{abstract}
Raney nickel in refluxing 2-propanol is an effective catalytic system for cleaving $\mathrm{C}-\mathrm{O}$ bonds in aromatic alcohols by transfer hydrogenolysis. Deoxygenation of alcohols substituted at the $\alpha-, \beta-, \gamma-, \delta$-, and $\varepsilon$-positions was accomplished. The reaction appears not to be sensitive to substitution about the carbinol carbon. Aliphatic alcohols do not undergo hydrogenolysis with this system. Some dehydromethylation is found in the hydrogenolysis of primary alcohols. With extended reaction times, ring reduction accompanies hydrogenolysis of alcohols containing more than one aromatic ring. Raney cobalt is shown to catalyze hydrogen transfer from 2-propanol. Raney cobalt in refluxing 2-propanol is an effective system for deoxygenating $\alpha$-substituted alcohols only. Although Raney cobalt is less reactive than Raney nickel in transfer hydrogenolysis, it exhibits greater selectivity as illustrated by the lack of ring reduction in alcohols containing more than one aromatic ring. () 2001 Elsevier Science B.V. All rights reserved.
\end{abstract}

Keywords: Raney nickel; Raney cobalt; Catalytic transfer hydrogenolysis; Hydrogen donor; Deoxygenation of aromatic alcohols

\section{Introduction}

Raney nickel is widely recognized as a versatile catalyst for effecting reductive transformations of organic compounds [1,2]. Less well known and utilized is Raney nickel's ability to catalyze reductions using hydrogen donors instead of molecular hydrogen $[3,4]$. Known as catalytic transfer hydrogenation, this remarkable reaction was first described 50 years ago by Kleiderer and Kornfeld [5] in their study on the Raney nickel catalyzed transfer of hydrogen from cholesterol to cyclohexanone. Since the first report, Raney nickel has been shown to catalyze the transfer

\footnotetext{
* Corresponding author. Tel.: +1-423-755-4709; fax: +1-423-755-5234.

E-mail address: robert-mebane@utc.edu (R.C. Mebane).

${ }^{1}$ Co-corresponding author.
}

of hydrogen from a variety of hydrogen donors $[3,4]$. 2-Propanol is a useful donor because of its simplicity, ready availability, and ease of use. Although the literature is somewhat sparse, Raney nickel catalyzed transfer hydrogenations utilizing 2-propanol have been reported for the reduction of olefins [6], ketones [6-8], phenols [6], aromatic nitro compounds [9-11], and certain aromatic hydrocarbons [6,12].

Our own interest in this area was piqued by the observation of Andrews and Pillai [6] that benzyl alcohol, benzhydrol and $\alpha$-tetralol can undergo hydrogenolysis with Raney nickel in refluxing 2-propanol. Catalytic hydrogenolysis of benzyl alcohols with molecular hydrogen has long been known [13]. Indeed, catalytic hydrogenolysis of $\mathrm{C}-\mathrm{O}$ bonds $\alpha$ to an aromatic ring in derivatives of benzyl alcohols has made the benzyl group a useful protecting group in multistep synthesis $[14,15]$. Interestingly, so 
far as we know, the hydrogenolysis of alcohols under hydrogen transfer conditions utilizing Raney nickel and 2-propanol has not been systematically studied. Furthermore, hydrogen transfer from 2-propanol with Raney cobalt has not been reported. Thus, as part of our continuing work on transfer hydrogenations with Raney catalysts and 2-propanol, we wish to describe how Raney nickel in refluxing 2-propanol efficiently deoxygenates aromatic alcohols under neutral conditions. In addition we wish to report that Raney cobalt does catalyze the transfer of hydrogen from 2-propanol and that the decreased reactivity of this catalyst is warranted in the hydrogenolysis of benzyl alcohols to suppress certain side reductions which can be encountered with Raney nickel. The mild conditions employed in these reactions offer considerable advantages over the conventional method of catalytic hydrogenolysis as neither hydrogen containment nor a pressure vessel is required.

\section{Experimental}

1,2-Diphenylethanol $\left(66.5-67.5^{\circ} \mathrm{C}\right.$, lit. $\mathrm{mp} 67^{\circ} \mathrm{C}$ [16]) and 1-(2-fluorenyl)ethanol (mp $138-139^{\circ} \mathrm{C}$, lit. $\mathrm{mp} 139-140^{\circ} \mathrm{C}$ [17]) were prepared by sodium borohydride reduction of 1,2-diphenylethanone and 2 -acetylfluorene, respectively. The remaining alcohols used in this study were available from commercial suppliers and were used as obtained unless impurities were detected by GC analysis in which case the alcohols were purified by distillation or recrystallization. All alcohols used in this study were found by GC analysis to have a purity in excess of $98 \%$. Progress of the hydrogenolysis reactions was monitored by GC-MS using a fused silica capillary column (methyl $50 \%$ phenyl silicone, $25 \mathrm{~m} \times 0.25 \mathrm{~mm}$ i.d., $0.25 \mu \mathrm{m}$ film thickness). With the exception of those that follow, the products were identified by comparison of retention times and fragmentation patterns with authentic samples. 2-Ethylfluorene [18], 5,6,7,8-tetrahydro-2-ethylnaphthalene [19], 1,2, 3,4-tetrahydro-2-ethylnaphthalene [19], 5,6,7,8-tetrahydro-1-ethylnaphthalene [19], 1-cyclohexyl-2-phenylethane [20], and cis-hexahydrofluoren-9-one [21] were found to have physical or spectral properties identical to published reports. Raney ${ }^{\circledR} 2800$ nickel and Raney ${ }^{\circledR} 2700$ cobalt were obtained from W.R.
Grace Company, Chattanooga Davison. The Raney ${ }^{\circledR}$ 2800 nickel has a BET surface area of $82 \mathrm{~m}^{2} / \mathrm{g}$ and a particle size range of $45-90 \mathrm{~mm}$ [22]. Raney ${ }^{\circledR} 2700$ cobalt has a BET surface area of $12 \mathrm{~m}^{2} / \mathrm{g}$ and a particle size range of 20-50 $\mathrm{mm}$ [22]. The Raney catalysts were washed prior to use with distilled water (six times) and 2-propanol (three times) and stored in 2-propanol.

CAUTION: Raney nickel is a pyrophoric solid when dry and may ignite spontaneously in air.

\subsection{General procedure for Raney nickel catalyzed hydrogenolysis of aromatic alcohols}

The alcohol ( $2 \mathrm{~g})$ was added to a mixture of Raney nickel $(5 \mathrm{~g})$ in 2-propanol $(30 \mathrm{ml})$. While open to the atmosphere, the reaction mixture was vigorously stirred and refluxed (water-cooled condenser attached to flask) for the times indicated for the individual alcohols listed in Tables 1-3. Aliquots were removed at $0.25 \mathrm{~h}$ intervals and analyzed by GC-MS. The yields reported in Tables 1-3 represent percentage conversion of the starting alcohol to reduced product as determined by peak areas and are the average of at least two reactions. Isolation of the reduced product involved decanting the 2-propanol solution, washing the Raney nickel with 2-propanol $(3 \times 10 \mathrm{ml})$, filtering the combined 2-propanol layers through celite, and evaporation of the 2-propanol and acetone.

\subsection{General procedure for Raney cobalt catalyzed hydrogenolysis of aromatic alcohols}

This procedure was identical to that described above for Raney nickel except that $4 \mathrm{~g}$ of Raney cobalt were used in the reductions.

\section{Results and discussion}

All of the aromatic alcohols used in this study with the exception of 1,2-diphenylethanol and 1-(2-fluorenyl)ethanol were available from commercial suppliers. 1,2-Diphenylethanol and 1-(2-fluorenyl) ethanol were conveniently prepared by sodium borohydride reduction of 1,2-diphenylethanone and 2acetylfluorene, respectively (see Section 2). Tables 1-3 
Table 1

Raney nickel and Raney cobalt catalyzed transfer hydrogenolysis of benzylic alcohols with 2-propanol (one aromatic ring)

\begin{tabular}{|c|c|c|c|c|c|}
\hline Entry & Substrate & Raney catalyst & Time (h) & Product(s) & Yield $(\%)$ \\
\hline \multirow[t]{4}{*}{1} & \multirow[t]{4}{*}{ Benzyl alcohol } & \multirow[t]{3}{*}{$\mathrm{Ni}$} & \multirow[t]{3}{*}{1.0} & Toluene & 87 \\
\hline & & & & Benzene & 7 \\
\hline & & & & Benzaldehyde & 4 \\
\hline & & $\mathrm{Co}$ & 3.0 & Toluene & 95 \\
\hline \multirow[t]{3}{*}{2} & \multirow[t]{3}{*}{ 4-Isopropylbenzyl alcohol } & \multirow[t]{2}{*}{$\mathrm{Ni}$} & \multirow[t]{2}{*}{0.25} & 4-Isopropyltoluene & 90 \\
\hline & & & & Isopropylbenzene & 10 \\
\hline & & $\mathrm{Co}$ & 24 & 4-Isopropyltoluene & $8^{a}$ \\
\hline \multirow[t]{4}{*}{3} & \multirow[t]{4}{*}{ 4-Methoxybenzyl alcohol } & \multirow[t]{3}{*}{$\mathrm{Ni}$} & \multirow[t]{3}{*}{0.25} & 4-Methoxytoluene & 88 \\
\hline & & & & Methoxybenzene & 9 \\
\hline & & & & Toluene & 2 \\
\hline & & Co & 24 & 4-Methoxytoluene & $35^{\mathrm{a}}$ \\
\hline \multirow[t]{2}{*}{4} & \multirow[t]{2}{*}{ 1-Phenylethanol } & $\mathrm{Ni}$ & 0.25 & Ethylbenzene & 96 \\
\hline & & $\mathrm{Co}$ & 3.0 & Ethylbenzene & 100 \\
\hline \multirow[t]{2}{*}{5} & \multirow[t]{2}{*}{ 1-( $p$-Tolyl)ethanol } & $\mathrm{Ni}$ & 0.50 & 4-Ethyltoluene & 99 \\
\hline & & Co & 24 & 4-Ethyltoluene & 96 \\
\hline \multirow[t]{5}{*}{6} & \multirow[t]{5}{*}{ 1-(4-Methoxyphenyl)ethanol } & \multirow[t]{4}{*}{$\mathrm{Ni}$} & \multirow[t]{2}{*}{0.25} & 4-Methoxyethylbenzene & 94 \\
\hline & & & & Ethylbenzene & 6 \\
\hline & & & \multirow[t]{2}{*}{6.0} & 4-Methoxyethylbenzene & 46 \\
\hline & & & & Ethylbenzene & 54 \\
\hline & & Co & 24 & 4-Methoxyethylbenzene & 92 \\
\hline \multirow[t]{2}{*}{7} & \multirow[t]{2}{*}{ 1-Phenyl-1-butanol } & $\mathrm{Ni}$ & 0.25 & Butylbenzene & 98 \\
\hline & & Co & 1.0 & Butylbenzene & 100 \\
\hline \multirow[t]{2}{*}{8} & \multirow[t]{2}{*}{ 1-Phenyl-1-pentanol } & $\mathrm{Ni}$ & 0.25 & Pentylbenzene & 100 \\
\hline & & Co & 2.0 & Pentylbenzene & 100 \\
\hline \multirow[t]{2}{*}{9} & \multirow[t]{2}{*}{ 2,2-Dimethyl-1-phenyl-1-propanol } & $\mathrm{Ni}$ & 4.0 & 2,2-Dimethyl-1-phenylpropane & 100 \\
\hline & & Co & 24 & 2,2-Dimethyl-1-phenylpropane & $9^{\mathrm{a}}$ \\
\hline \multirow[t]{2}{*}{10} & \multirow[t]{2}{*}{ Ethyl mandelate } & $\mathrm{Ni}$ & 1.0 & Ethyl phenylacetate & 100 \\
\hline & & Co & 8.0 & Ethyl phenylacetate & 98 \\
\hline \multirow[t]{2}{*}{11} & 2-Phenyl-2-propanol & $\mathrm{Ni}$ & 0.25 & Isopropylbenzene & 100 \\
\hline & & Co & 3.5 & Isopropylbenzene & 99 \\
\hline 12 & 1-Phenyl-1-cyclohexanol & $\mathrm{Ni}$ & 0.25 & Cyclohexylbenzene & 100 \\
\hline & & Co & 7.0 & Cyclohexylbenzene & 99 \\
\hline
\end{tabular}

${ }^{a}$ Remainder is starting material.

summarize the 31 aromatic alcohols investigated in this study. The experimental procedure for the transfer hydrogenolysis reaction is simple and straightforward. To illustrate, the alcohol is stirred magnetically with a suspension Raney catalyst in refluxing 2-propanol while open to the atmosphere. The substrate to catalyst ratio was $2: 5$ by weight for Raney nickel and 2:4 by weight for Raney cobalt. The catalyst loading for Raney nickel is comparable to that used by others reporting on hydrogen transfer reactions $[11,12,23]$.
As described later, we find that the catalytic activity of the Raney catalysts is retained after repeated use. The progress of the reactions was conveniently monitored by GC-MS. The reduced products were readily isolated after filtration through celite to remove the Raney catalyst followed by solvent removal. Products were identified whenever possible by comparison of retention times and fragmentation patterns with authentic samples or by comparison with published physical and spectral data (see Section 2). 
Table 2

Raney nickel and Raney cobalt catalyzed transfer hydrogenolysis of benzylic alcohols with 2-propanol (two or more aromatic rings)

\begin{tabular}{|c|c|c|c|c|c|}
\hline Entry & Substrate & Raney catalyst & Time (h) & Product(s) & Yield $(\%)$ \\
\hline \multirow[t]{3}{*}{1} & \multirow[t]{3}{*}{ 1,2-Diphenylethanol } & $\mathrm{Ni}$ & 1.0 & Bibenzyl & 97 \\
\hline & & & 5.0 & 1-Cyclohexyl-2-phenylethane & 99 \\
\hline & & Co & 1.5 & Bibenzyl & 100 \\
\hline \multirow[t]{4}{*}{2} & \multirow[t]{4}{*}{ Benzhydrol } & $\mathrm{Ni}$ & 0.25 & Diphenylmethane & 91 \\
\hline & & & & Cyclohexylphenylmethane & 4 \\
\hline & & & 10 & Cyclohexylphenylmethane & 96 \\
\hline & & Co & 1.0 & Diphenylmethane & 100 \\
\hline \multirow[t]{5}{*}{3} & \multirow[t]{5}{*}{ Triphenylmethanol } & $\mathrm{Ni}$ & 0.25 & Triphenylmethane & 96 \\
\hline & & & & Diphenylcyclohexylmethane & 4 \\
\hline & & & 24 & Triphenylmethane & 65 \\
\hline & & & & Diphenylcyclohexylmethane & 33 \\
\hline & & Co & 24 & Triphenylmethane & $80^{\mathrm{a}}$ \\
\hline \multirow[t]{4}{*}{4} & \multirow[t]{4}{*}{ 9-Hydroxyfluorene } & $\mathrm{Ni}$ & 1.0 & Fluorene & 33 \\
\hline & & & & Hexahydro-9-fluorenone & 67 \\
\hline & & & 24 & Complex mixture $^{\mathrm{b}}$ & \\
\hline & & Co & 0.75 & Fluorene & 100 \\
\hline \multirow[t]{2}{*}{5} & \multirow[t]{2}{*}{ Dibenzosuberenol } & $\mathrm{Ni}$ & 0.50 & Dibenzosuberane & 100 \\
\hline & & Co & 1.0 & Dibenzosuberene & 93 \\
\hline \multirow[t]{4}{*}{6} & \multirow[t]{4}{*}{ 1-(2-Fluorenyl)ethanol } & $\mathrm{Ni}$ & 1.0 & 2-Ethylfluorene & 95 \\
\hline & & & 4.0 & 2-Ethylfluorene & 64 \\
\hline & & & & Ring reduced products ${ }^{c}$ & 36 \\
\hline & & Co & 2.0 & 2-Ethylfluorene & 100 \\
\hline \multirow[t]{3}{*}{7} & \multirow[t]{3}{*}{ 1-(1-Naphthyl)ethanol } & $\mathrm{Ni}$ & 4.0 & 5,6,7,8-Tetrahydro-1-ethylnaphthalene & 84 \\
\hline & & & & 1,2,3,4-Tetrahydro-1-ethylnaphthalene & 16 \\
\hline & & Co & 0.75 & 1-Ethylnaphthalene & 100 \\
\hline \multirow[t]{3}{*}{8} & \multirow[t]{3}{*}{ 1-(2-Naphthyl)ethanol } & $\mathrm{Ni}$ & 4.0 & 5,6,7,8-Tetrahydro-2-ethylnaphthalene & 82 \\
\hline & & & & 1,2,3,4-Tetrahydro-2-ethylnaphthalene & 14 \\
\hline & & Co & 0.50 & 2-Ethylnaphthalene & 100 \\
\hline \multirow[t]{3}{*}{9} & \multirow[t]{3}{*}{ 1-(4-Biphenylyl)ethanol } & $\mathrm{Ni}$ & 0.50 & 4-Ethylbiphenyl & $84^{\mathrm{d}}$ \\
\hline & & & 24 & Ring reduced products ${ }^{\mathrm{e}}$ & \\
\hline & & Co & 5.0 & 4-Ethylbiphenyl & 98 \\
\hline
\end{tabular}

\footnotetext{
${ }^{\text {a }}$ Remainder is starting material.

${ }^{\mathrm{b}} \mathrm{GC}-\mathrm{MS}$ suggests mostly hexahydrofluorene.

${ }^{c}$ GC-MS suggests a 1:1 mixture of 2-ethyl- and 7-ethyl-2,3,4,4 $\alpha, 9,9 \alpha$-hexahydrofluorene.

${ }^{\mathrm{d}} \mathrm{GC}-\mathrm{MS}$ suggests that remainder is 1-(4-cyclohexylphenyl)ethanol.

${ }^{\mathrm{e}} \mathrm{GC}-\mathrm{MS}$ and ${ }^{1} \mathrm{H}$ NMR suggests a nearly 1:1 mixture of 1-cyclohexyl-4-ethylbenzene and trans-1-ethyl-4-phenylcyclohexane.
}

\subsection{Raney nickel reductions}

Aromatic alcohols are readily deoxygenated by transfer hydrogenolysis with Raney nickel and refluxing 2-propanol as seen in Tables 1-3. The hydrogenolysis reaction is generally complete in a matter of a few minutes to a few hours. The yields reported in Tables 1-3 represent percentage conversion of starting alcohol as determined by GC.
For the alcohols 1-phenyl-1-cyclohexanol, 1-phenyl-1pentanol, and dibenzosuberenol the isolated yields of the hydrogenolysis products were 91, 80 and 94\%, respectively.

As evidenced by the reaction times reported in Table 1, secondary and tertiary benzyl alcohols containing a single aromatic ring (entries 4-12) undergo rapid hydrogenolysis with Raney nickel and 2-propanol to give alkylbenzenes in excellent yields. 
Table 3

Raney nickel and Raney cobalt catalyzed transfer hydrogenolysis of non-benzylic aromatic alcohols with 2-propanol

\begin{tabular}{|c|c|c|c|c|c|}
\hline Entry & Substrate & Raney catalyst & Time (h) & Product(s) & Yield $(\%)$ \\
\hline \multirow[t]{3}{*}{1} & \multirow[t]{3}{*}{ 2-Phenylethanol } & $\mathrm{Ni}$ & 3.0 & Ethylbenzene & 80 \\
\hline & & & & Toluene & 17 \\
\hline & & $\mathrm{Co}$ & 24 & Ethylbenzene & $17^{\mathrm{a}}$ \\
\hline \multirow[t]{2}{*}{2} & \multirow[t]{2}{*}{ 1-Phenyl-2-propanol } & $\mathrm{Ni}$ & 0.50 & Propylbenzene & 100 \\
\hline & & $\mathrm{Co}$ & 24 & Propylbenzene & $34^{\mathrm{a}}$ \\
\hline \multirow[t]{3}{*}{3} & \multirow[t]{3}{*}{ 1-Phenyl-2-butanol } & $\mathrm{Ni}$ & 0.50 & Butylbenzene & 100 \\
\hline & & $\mathrm{Co}$ & 8.0 & Butylbenzene & $17^{\mathrm{a}}$ \\
\hline & & & 24 & Butylbenzene & $41^{\mathrm{a}}$ \\
\hline \multirow[t]{2}{*}{4} & \multirow[t]{2}{*}{ 2-Methyl-1-phenyl-2-propanol } & $\mathrm{Ni}$ & 0.75 & Isobutylbenzene & 98 \\
\hline & & $\mathrm{Co}$ & 24 & Isobutylbenzene & $<2^{\mathrm{a}}$ \\
\hline \multirow[t]{3}{*}{5} & \multirow[t]{3}{*}{ 3-Phenyl-1-propanol } & $\mathrm{Ni}$ & 2.0 & Propylbenzene & 80 \\
\hline & & & & Ethylbenzene & 20 \\
\hline & & $\mathrm{Co}$ & 24 & No reaction & \\
\hline \multirow[t]{2}{*}{6} & \multirow[t]{2}{*}{ 4-Phenyl-2-butanol } & $\mathrm{Ni}$ & 3.0 & Butylbenzene & 98 \\
\hline & & $\mathrm{Co}$ & 24 & No reaction & \\
\hline \multirow[t]{2}{*}{7} & \multirow[t]{2}{*}{ 2-Methyl-4-phenyl-2-butanol } & $\mathrm{Ni}$ & 0.75 & Isopentylbenzene & 100 \\
\hline & & $\mathrm{Co}$ & 24 & No reaction & \\
\hline \multirow[t]{3}{*}{8} & \multirow[t]{3}{*}{ 4-Phenyl-1-butanol } & $\mathrm{Ni}$ & 10 & Butylbenzene & 67 \\
\hline & & & & Propylbenzene & 33 \\
\hline & & $\mathrm{Co}$ & & No reaction & \\
\hline \multirow[t]{2}{*}{9} & \multirow[t]{2}{*}{ 5-Phenyl-2-pentanol } & $\mathrm{Ni}$ & 7.0 & Pentylbenzene & $95^{\mathrm{b}}$ \\
\hline & & $\mathrm{Co}$ & 24 & No reaction & \\
\hline \multirow[t]{3}{*}{10} & \multirow[t]{3}{*}{ 5-Phenyl-1-pentanol } & $\mathrm{Ni}$ & 6.0 & Pentylbenzene & 81 \\
\hline & & & & Butylbenzene & 19 \\
\hline & & Co & 24 & No reaction & \\
\hline
\end{tabular}

${ }^{a}$ Remainder is starting material.

${ }^{\mathrm{b}}$ The MS of the remainder is consistent with 5-cyclohexyl-2-pentanol.

Hydrogenolysis of both the hydroxyl group and the methoxy group occurs in the Raney nickel catalyzed reaction of 1-(4-methoxyphenyl)ethanol (Table 1, entry 3). Loss of the hydroxyl group is much faster than the hydrogenolysis of the methoxy group. Thus, 15 min into the reaction all of the starting alcohol is consumed and 4-methoxyethylbenzene, the expected product of alcohol hydrogenolysis, is the major product $(88 \%)$. If the reaction is allowed to proceed for a longer time, then hydrogenolysis of the methoxy group to give ethylbenzene becomes significant.

In addition to the expected hydrogenolysis products, the three primary benzyl alcohols used in this study (Table 1, entries 1-3) give to a small extent deoxygenated products containing one less carbon. Andrews and Pillai [6] observed a similar result in their Raney nickel study with benzyl alcohol. This dehydromethylation reaction of primary alcohols with nickel catalyst is not without precedence. For example, dehydromethylation of primary alcohols has been observed with $\mathrm{Ni} / \mathrm{Al}_{2} \mathrm{O}_{3}$ catalyst [24] and with Raney nickel in refluxing toluene $[23,25]$. The most likely origin of this dehydromethylation side reaction involves the reversible nickel catalyzed oxidation of the primary alcohol to an aldehyde followed by a decarbonylation step which is well known [26].

As seen in Table 2, benzyl alcohols containing more than one aromatic ring undergo hydrogenolysis with Raney nickel in refluxing 2-propanol. In addition, prolonged reaction times can lead to ring reduction by transfer hydrogenation. To illustrate, hydrogenolysis of 1,2-diphenylethanol (Table 2, entry 
1) to give bibenzyl is essentially complete after $1 \mathrm{~h}$ of reflux. The reaction can be stopped at this stage and the bibenzyl conveniently isolated. If, however, the refluxing is continued beyond $1 \mathrm{~h}$, hydrogenation of one of the aromatic rings in bibenzyl is observed. Essentially all of the bibenzyl is reduced to 1-cyclohexyl-2-phenylethane in $5 \mathrm{~h}$.

Hydrogenolysis of benzyhydrol and triphenylmethanol (Table 2, entries 2 and 3 ) is rapid and essentially complete after $15 \mathrm{~min}$ giving diphenylmethane and triphenylmethane, respectively. Further conversion of diphenylmethane into cyclohexylphenylmethane by single ring reduction is quite good and complete within $10 \mathrm{~h}$. Single ring reduction of triphenylmethane appears to occur much slower and is probably due to increased steric hindrance caused by the third ring which prevents the molecule from effectively adsorbing to the surface of the catalyst.

In addition to rapid hydrogenolysis, the vinyl bond in dibenzosuberenol (Table 2, entry 5) undergoes hydrogenation to give dibenzosuberane as the final product. Both reductions are complete within $30 \mathrm{~min}$. In contrast to the other alcohol examples in Table 2, ring reduction in dibenzosuberane is extremely slow with $<10 \%$ being detected after $24 \mathrm{~h}$ of reflux. The lack of ring reduction in dibenzosuberane is probably due more to a conformational effect and not a steric effect. As described in more detail below, we believe that ring reduction in our polycyclic systems results from assisted adsorption of one of the aromatic rings on the catalyst surface which brings other rings in proximity to the hydrogenation sites on the catalyst. A molecular model of the preferred conformation of dibenzosuberane shows that the two aromatic rings are far from coplanar. The model further suggests that effective adsorption of one of the rings on the catalyst surface causes the second ring to be directed away from the catalyst surface.

The reaction of 9-hydroxyfluorene (Table 2, entry 4) with Raney nickel in refluxing 2-propanol is complete after $1 \mathrm{~h}$. Oxidation and ring reduction to give cis-hexahydrofluoren-9-one is favored 2 to 1 over hydrogenolysis which yields fluorene. A complex mixture containing mostly hexahydrofluorene is obtained if the reaction is allowed to proceed for $24 \mathrm{~h}$.

Deoxygenation of 1-(2-fluorenyl)ethanol (Table 2, entry 6) is essentially complete after $1 \mathrm{~h}$ giving 2-ethylfluorene as the only product. Ring reduction of the 2-ethylfluorene is observed with prolonged refluxing.

Hydrogenolysis of the two isomeric naphthyl-1ethanols (Table 2, entries 7 and 8) was not as clean as the previous examples due to the rapid hydrogenation of one of the naphthyl rings. To illustrate, in the first few minutes of the Raney nickel catalyzed reaction of 1-(1-naphthyl)ethanol a mixture consisting of 1-ethylnaphthalene, starting material, and the two possible tetrahydronaphthalenes is detected. After $4 \mathrm{~h}$ of reflux two products, 5,6,7,8-tetrahydro-1-ethylnaphthalene and 1,2,3,4-tetrahydro-1-ethylnaphthalene, are detected with the former product predominating.

The results of our Raney nickel catalyzed hydrogenolysis of alcohols other than benzyl alcohols are summarized in Table 3. In this study we looked at the Raney nickel catalyzed hydrogenolysis of alcohols containing the hydroxyl group in the $\beta-, \gamma-, \delta$, and $\varepsilon$-position relative to the aromatic ring. We found that hydrogenolysis of secondary and tertiary alcohols in this group (Table 3, entries 2-4 and 6, 7 and 9) proceeds smoothly to give alkylbenzenes essentially quantitatively. As noted by the reaction times, the hydrogenolysis of these alcohols is generally slower than for the hydrogenolysis of benzyl alcohols in Tables 1 and 2. Furthermore, the time required for complete hydrogenolysis generally increases as the hydroxyl group moves farther away from the aromatic ring. As was observed with the primary benzyl in Table 1, some dehydromethylation occurs simultaneously with the hydrogenolysis reaction of the primary alcohols found in Table 3 (entries 1, 5, 8 and 10).

Raney nickel is known to contain adsorbed hydrogen which is formed in the activation of the catalyst. One estimate places the amount of adsorbed hydrogen per gram of catalyst at $2-5 \mathrm{mmol}[3,27]$. By reducing ethyl trans-cinnamate to the ethyl ester of 3-phenylpropanoic acid with Raney nickel in 2-propanol at room temperature we determined that our Raney catalyst contains $1.2 \mathrm{mmol} / \mathrm{g}$ of available hydrogen. ${ }^{2}$ To show that the Raney nickel used in this study does play a catalytic role in the oxidation and transfer of hydrogen from 2-propanol we subjected 1-phenylethanol to reductions in which the Raney nickel was reused after being washed

\footnotetext{
${ }^{2}$ Our experience suggests that the transfer of hydrogen from 2-propanol occurs more readily at elevated temperatures.
} 


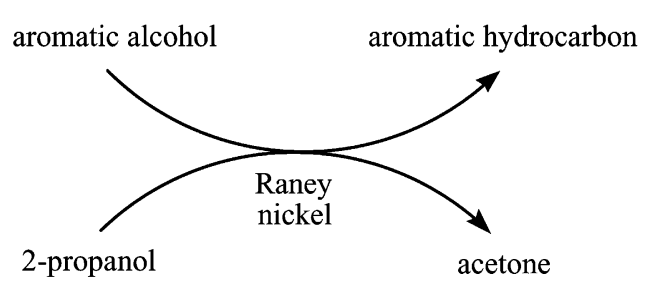

Fig. 1. Catalytic transfer hydrogenolysis.

with 2-propanol. We found that the catalyst retained activity through the seven reductions which were performed. In each reduction the alcohol was completely converted into ethylbenzene within a $15 \mathrm{~min}$ period. These results clearly establish that transfer of hydrogen from 2-propanol occurs.

The catalytic cycle depicted in Fig. 1 describes the overall process that takes place in our hydrogenolysis reactions. While the mechanism by which Raney nickel catalyzes the transfer of hydrogen from 2-propanol remains ambiguous, it is generally thought that heterogeneous catalytic hydrogen transfer reactions are not simply conventional catalytic hydrogenations with a donor molecule providing the necessary hydrogen [3]. Although this study does not add directly to the mechanism of catalytic transfer hydrogenation, it appears that the facile $\mathrm{C}-\mathrm{O}$ bond cleavage observed in our reactions is the result of adsorption of the aromatic ring on the nickel surface which then brings the $\mathrm{C}-\mathrm{O}$ bond into close proximity with the active site on the catalyst used for bond cleavage. Supporting this notion of assisted adsorption is our finding that the inclusion of benzene (20 vol.\%) in the Raney nickel/2-propanol hydrogenolysis of 1-phenyl-2-propanol leads to a four-fold increase in the time required to completely deoxygenate the compound. In this experiment, benzene competitively adsorbs on the nickel surface blocking sites for adsorption by the 1-phenyl-2-propanol.

Further support for assisted adsorption comes from our observation that aliphatic alcohols do not undergo hydrogenolysis when subjected to the same reaction conditions. The nine aliphatic alcohols we used in this part of the study were 3-methyl-3-octanol, 4-t-butylcyclohexanol, 1-propylcyclohexanol, 8-hydroxy-p-menthane, 3-octanol, 2-octanol, 1-dodecanol, 1-tetradecanol, and 1-octadecanol. No reaction of any kind occurred with the first four alcohols after $24 \mathrm{~h}$ of reflux with Raney nickel and 2-propanol. Within a $24 \mathrm{~h}$ period 2-octanol and 3-octanol did undergo oxidation to the corresponding ketones to a small extent $(<10 \%)$. Interestingly, 1-dodecanol, 1-tetradecanol, and 1-octadecanol do undergo dehydromethylation under the same reaction conditions. The yields of undecane, tridecane and heptadecane were 7, 16 and $31 \%$, respectively.

As previously described, prolonged reaction times in the hydrogenolysis of alcohols containing more than one aromatic ring can lead to ring reduction of the hydrogenolysis product. This transfer hydrogenation is likely facilitated by assisted adsorption of one of the aromatic rings on the nickel surface in a similar manner to that described above for hydrogenolysis. In their comprehensive study of hydrogen transfer reactions with Raney nickel, Andrews and Pillai [6] observed ring reduction of polycyclic aromatic rings and further found that only one ring is reduced in diphenyl systems. They suggest in their study that one of the phenyl rings adsorbs on a non-active site, such as alumina, which brings the other ring in close proximity to the hydrogenation site.

The hydrogenolysis of benzyl alcohols under conventional catalytic hydrogenation conditions using palladium has been shown to be sensitive to substitution around the carbinol carbon with the ease of the cleavage decreasing with increased substitution [28]. We have been unable to find a comparable study in the literature describing steric effects in the hydrogenolysis of benzyl alcohols with Raney nickel. Although we anticipated that the hydrogenolysis of alcohols by hydrogen transfer with Raney nickel would be sensitive to steric hindrance about the carbinol position, our results do not bear this out. As seen in Tables 1 and 2, tertiary benzyl alcohols (Table 1, entries 11 and 12 and Table 2, entry 3) undergo hydrogenolysis as easily as secondary and primary benzyl alcohols with these reactions requiring just minutes to reach completion. The one exception is 2,2-dimethyl-1-phenyl-1-propanol (Table 1 , entry 9) which requires $4 \mathrm{~h}$ to reach completion. Most likely the decreased reactivity of this alcohol is the result of the $t$-butyl group hindering the effective adsorption of the phenyl and alcohol groups onto the catalyst surface. Steric effects also appear to be absent in the hydrogenolysis of aromatic alcohols other than benzyl alcohols as 
evidenced by the facile $\mathrm{C}-\mathrm{O}$ cleavage observed in the reactions of 2-methyl-1-phenyl-2-propanol and 2-methyl-4-phenyl-2-butanol (Table 3, entries 4 and 7). Further work is underway to better understand the lack of steric effects in these reactions.

\subsection{Raney cobalt reductions}

In contrast to Raney nickel, Raney cobalt is seldom used in catalytic hydrogenation reactions. This may be due in part to the fact that Raney cobalt is less reactive than Raney nickel [29]. As far as we know, there are no reports in the literature describing the use of Raney cobalt as a catalyst for transfer hydrogenations. In our present work we hoped to demonstrate that Raney cobalt can indeed catalyze hydrogen transfer from 2-propanol and that this reaction could be used in the hydrogenolysis of aromatic alcohols. Furthermore, we hoped to capitalize on Raney cobalt's decreased reactivity to minimize the side reactions, such as ring reduction and dehydromethylation, encountered in some of the Raney nickel hydrogenolysis reactions discussed previously.

As expected, Raney cobalt does facilitate hydrogen transfer from 2-propanol with concomitant cleavage of the $\mathrm{C}-\mathrm{O}$ bond in benzyl alcohols. As seen in Tables 1 and 2, Raney cobalt hydrogenolysis gives excellent yields of alkylbenzenes for most benzyl alcohols. For alcohols 1,2-diphenylethanol, 1-(2-naphthyl)ethanol, and 1-(2-fluorenyl)ethanol the isolated yields of the hydrogenolysis products were 94,95 and $97 \%$, respectively. The origin of the poor yields obtained with 4-isopropylbenzyl alcohol and 4-methoxybenzyl alcohol (Table 1, entries 2 and 3) is not obvious to us, particularly since the hydrogenolysis of benzyl alcohol and 1-(4-methoxyphenyl)ethanol (Table 1, entries 1 and 6) is nearly complete. It may be due to an electronic effect. It has been shown that under conventional catalytic hydrogenation conditions with molecular hydrogen, both Raney nickel and palladium catalyzed hydrogenolysis of ring substituted benzyl alcohols is sensitive to the electronic nature of the ring substituent $[28,30]$. The unreactivity of 2,2-dmethyl-1-phenyl-1-propanol (Table 1, entry 9) is most likely due to steric hindrance from the $t$-butyl group.

Although hydrogenolysis with Raney cobalt is slower than with Raney nickel, no dehydromethy- lation of benzyl alcohol is observed with Raney cobalt (Table 1, entry 1). In addition, Raney cobalt hydrogenolysis of 1-(4-methoxyphenyl)ethanol cleaves only the benzyl $\mathrm{C}-\mathrm{O}$ bond and leaves the 4-methoxy group untouched (Table 1, entry 6). The increased selectivity of Raney cobalt is also noteworthy in Table 2 where one finds no ring reduction accompanying hydrogenolysis of the eight alcohols containing two or more aromatic rings.

While Raney cobalt is effective at deoxygenating certain benzyl alcohols, it is not reactive enough to cleave $\mathrm{C}-\mathrm{O}$ bonds beyond the benzylic position. As seen in Table 3 , some hydrogenolysis of $\beta$-aryl alcohols (entries 1-4) is observed after long reaction times. No hydrogenolysis occurs for $\gamma$-, $\delta$-, or $\varepsilon$-aryl alcohols.

To show that the Raney cobalt plays a catalytic role in the oxidation and transfer of hydrogen from 2-propanol we subjected 1-phenylethanol to reductions in which the Raney cobalt was reused after being washed with 2-propanol. It was found that the catalyst retained activity through the six reductions which were performed. In each reduction the alcohol was completely converted into ethylbenzene within a $3 \mathrm{~h}$ period. These results clearly establish that transfer of hydrogen from 2-propanol occurs.

\section{Conclusion}

Raney nickel in refluxing 2-propanol readily cleaves $\mathrm{C}-\mathrm{O}$ bonds in aromatic alcohols by catalytic transfer hydrogenolysis. This reaction should be particularly useful in deoxygenating secondary and tertiary alcohols including alcohols where the hydroxyl group is located some distance from the aromatic ring. The reaction appears not to be sensitive to substitution around the carbinol carbon. This method has the advantage of not requiring the handling of gaseous hydrogen and involves a convenient workup consisting of filtration and solvent removal. The synthetic utility of the reaction may be diminished for primary aromatic alcohols as dehyromethylation accompanies the hydrogenolysis of primary aromatic alcohols. In addition, attention should be given to the deoxygenation of benzyl alcohols containing two or more aromatic rings since prolonged reaction times result in ring reduction products in addition to hydrogenolysis. 
In this work we have shown that Raney cobalt can catalyze the transfer of hydrogen from 2-propanol and that this hydrogen transfer can be used to deoxygenate $\alpha$-substituted aromatic alcohols. Raney cobalt is less reactive than Raney nickel and is only effective at deoxygenating $\alpha$-substituted alcohols. The decreased reactivity of Raney cobalt can be used to an advantage in that no ring reduction is encountered in the deoxygenation of $\alpha$-substituted alcohols containing two or more aromatic rings as can be the outcome with Raney nickel and prolonged reaction times.

\section{Acknowledgements}

The authors are grateful to the University of Chattanooga Foundation Grote Chemistry Fund for financial support of this work. In addition, we are indebted to W.R. Grace Company, Chattanooga Davison for the generous donation of Raney catalysts.

\section{References}

[1] H.O. House, Modern Synthetic Reactions, 2nd Edition, Benjamin, Menlo Park, 1972, p. 1.

[2] R.L. Augustine, Heterogeneous Catalysis for the Synthetic Chemist, Marcel Dekker, New York, 1996.

[3] G. Brieger, T.J. Nestrick, Chem. Rev. 74 (1974) 567.

[4] R.A.W. Johnstone, A.H. Wilby, I.D. Entwistle, Chem. Rev. 85 (1985) 129.

[5] E.C. Kleiderer, E.C. Kornfeld, J. Org. Chem. 13 (1948) 455

[6] M.J. Andrews, C.N. Pillai, Indian J. Chem. 16B (1978) 465.

[7] L.S. Stevovic, V. Soskic, I.O. Juranic, J. Serb. Chem. Soc. 60 (1995) 1071.

[8] E.M. Gonikberg, W.J. le Noble, J. Org. Chem. 60 (1995) 7751.

[9] A.A. Banerjee, D. Mukesh, J. Chem. Soc., Chem. Commun. (1988) 1275
[10] M. Chen, L. Kan, Huadong Huagong Xueyuan Xuebao 11 (1985) 105; Chem. Abstr. 103, 179938.

[11] E. Kuo, S. Srivastava, C.K. Cheung, W.J. le Noble, Synth. Commun. 15 (1985) 599.

[12] S. Srivastava, J. Minore, C.K. Cheung, W.J. le Noble, J. Org. Chem. 50 (1985) 394.

[13] M. Freifelder, Practical Catalytic Hydrogenation, Wiley, New York, 1971 (Chapter 19).

[14] W.H. Hartung, R. Simonoff, Org. React. 7 (1953) 263.

[15] T.W. Greene, Protective Groups in Organic Synthesis, Wiley, New York, 1981, pp. 29-30.

[16] G. Berti, F. Bottari, P.L. Ferrarini, B. Macchia, J. Org. Chem. 30 (1965) 4091.

[17] D.T. Mowry, M. Renoll, F.W. Huber, J. Am. Chem. Soc. 68 (1946) 1105.

[18] J. Buckingham (Ed.), Dictionary of Organic Compounds, 5th Edition, Suppl. 2, Chapman \& Hall, New York, 1984, p. 200.

[19] M. Adamczyk, D.S. Watt, D.A. Netzel, J. Org. Chem. 49 (1984) 4226.

[20] J.G. Grasselli, W.M. Ritchey (Eds.), Atlas of Spectral Data and Physical Constants for Organic Compounds, 2nd Edition, Vol. III, CRC Press, Cleveland, 1975, p. C1072.

[21] H.O. House, V. Paragamian, R.S. Ro, D.J. Wluka, J. Am. Chem. Soc. 82 (1960) 1457.

[22] Raney ${ }^{\circledR}$ Technical Manual, 4th Edition, W.R. Grace Company, Chattanooga Davison, TN, 1996, p. 21 and 23.

[23] M.E. Krafft, W.J. Crooks III, J. Org. Chem. 53 (1988) 432.

[24] W.F. Maier, I. Thies, P.V.R. Schleyer, Z. Naturforsch. 37B (1982) 392.

[25] M.E. Krafft, W.J. Crooks III, B. Zorc, S.E. Milczanowski, J. Org. Chem. 53 (1988) 3158.

[26] W.M. Schubert, R.R. Kintner, in: S. Patai (Ed.), The Chemistry of the Carbonyl Group, Wiley, New York, 1966, p. 749.

[27] R. Mozingo, D.E. Wolf, S.A. Harris, K. Folkers, J. Am. Chem. Soc. 65 (1943) 1013.

[28] A.P.G. Kieboom, J.F. de Kreuk, H. van Bekkum, J. Catal. 20 (1971) 58.

[29] R.L. Augustine, Heterogeneous Catalysis for the Synthetic Chemist, Marcel Dekker, New York, 1996, p. 216.

[30] Y. Oikawa, K. Tanka, O. Yonemitxu, Tetrahedron Lett. 25 (1984) 5397. 